\title{
Structural Effect of Carbon on Mn5Ge3 Thin Films Grown on Ge(001) Substrates by Solid Phase Epitaxy
}

Adriana Alvídrez-Lechuga ${ }^{1}$, Sion Olive-Méndez ${ }^{1}$, Luis Fuentes-Cobas ${ }^{2}$, José Holguín-Momaca ${ }^{1}$ and Jasper Plaisier ${ }^{3}$

${ }^{1}$ Centro de Investigación en Materiales Avanzados S.C., United States, ${ }^{2}$ Centro de Investigacion en Materiales Avanzados, United States, ${ }^{3}$ Eletttra - Sincrotrone Trieste, United States

Spintronic devices take advantage of the electron spin, considered as an additional degree of freedom. One of the challenges on the design of spintronic devices, as the spin-field effect transistor, is the achievement of spin injection into IV-group semiconductors via a Schottky barrier at room temperature (RT) [1]. Mn5Ge3 compound is the only FM phase with a Curie temperature (TC) of $296 \mathrm{~K}$, has been found that TC increases with carbon doping [2]. Mn5Ge3 has a hexagonal crystal structure P63/mcm and lattice constants $\mathrm{a}=7.184 \AA$ and $\mathrm{c}=5.053 \AA$, these characteristics allow the growth on $\mathrm{Ge}(111)$ substrates within a lattice mismatch of $3.7 \%$ [3]. However, $\mathrm{Ge}(111)$ is not compatible with the $\mathrm{Si}(001)$ technology, while $\mathrm{Ge}(001) / \mathrm{Mn} 5 \mathrm{Ge} 3$ heterostructures offer a good possibility for the design of spintronic devices. In this work, we present the growth of $\mathrm{Ge}(001) / \mathrm{Mn} 5 \mathrm{Ge} 3$ thin films using the solid phase epitaxy (SPE) method, samples doped with carbon atoms and without carbon were grown by magnetron-sputtering technique. Carbon doping has been found to affect the arrangement of the atomic structure. The SPE method consists of the deposition of $\mathrm{Mn}$ or co-deposition of $\mathrm{Mn}$ and $\mathrm{Ge}$ at room temperature (RT) followed by thermal annealing at Ts $=250{ }^{\circ} \mathrm{C}$ to induce $\mathrm{Ge}$ diffusion into the Mn layer to form the Mn5Ge3 layer.

Figure 1 shows the bi-dimensional grazing incidence x-ray diffraction (2D-GIXRD) pattern collected for a sample doped with carbon. The pattern shows discontinuous Debye rings that are associated with the texture, there are observed four peaks at 20.4, 28.3, 35.0, and 40.5 corresponding to the (102), (4-21), (5-21), and (5-12) reflections from the Mn5Ge3 compound. The reflection Ge(311) corresponds to the substrate. The indexation was based on the ICSD \#156103 crystallographic datasheet. Figure 2(a) shows the HRTEM micrograph for the sample without carbon, the c-axis of the Mn5Ge3 unit cell is normal to the $\mathrm{Ge}(001)$ plane of the substrate. The interface is relatively abrupt at the atomic scale along with a few atomic layers. The sample doped with carbon, figure 2(b), shows two stages of epitaxial growth: first, an Mn deposition, where the c-axis is normal to the $\mathrm{Ge}(001)$ plane, but the atomic arrangement is different from that in figure 2(a). In the Mn and Ge co-deposition stage, the c-axis of the Mn5Ge3 unit cell forms an angle with the plane of the substrate surface.

The authors thank the financial support obtained from Ciencia-Básica SEP-CONACYT grant No. 157559 and Fondo Mixto Chihuahua FOMIX grant No. CHIH-2011-C03-1688. The authors thank the beamtime at the beamline 7.1 MCX at Elettra Synchrotron to perform the 2D-GIXRD measurements. 


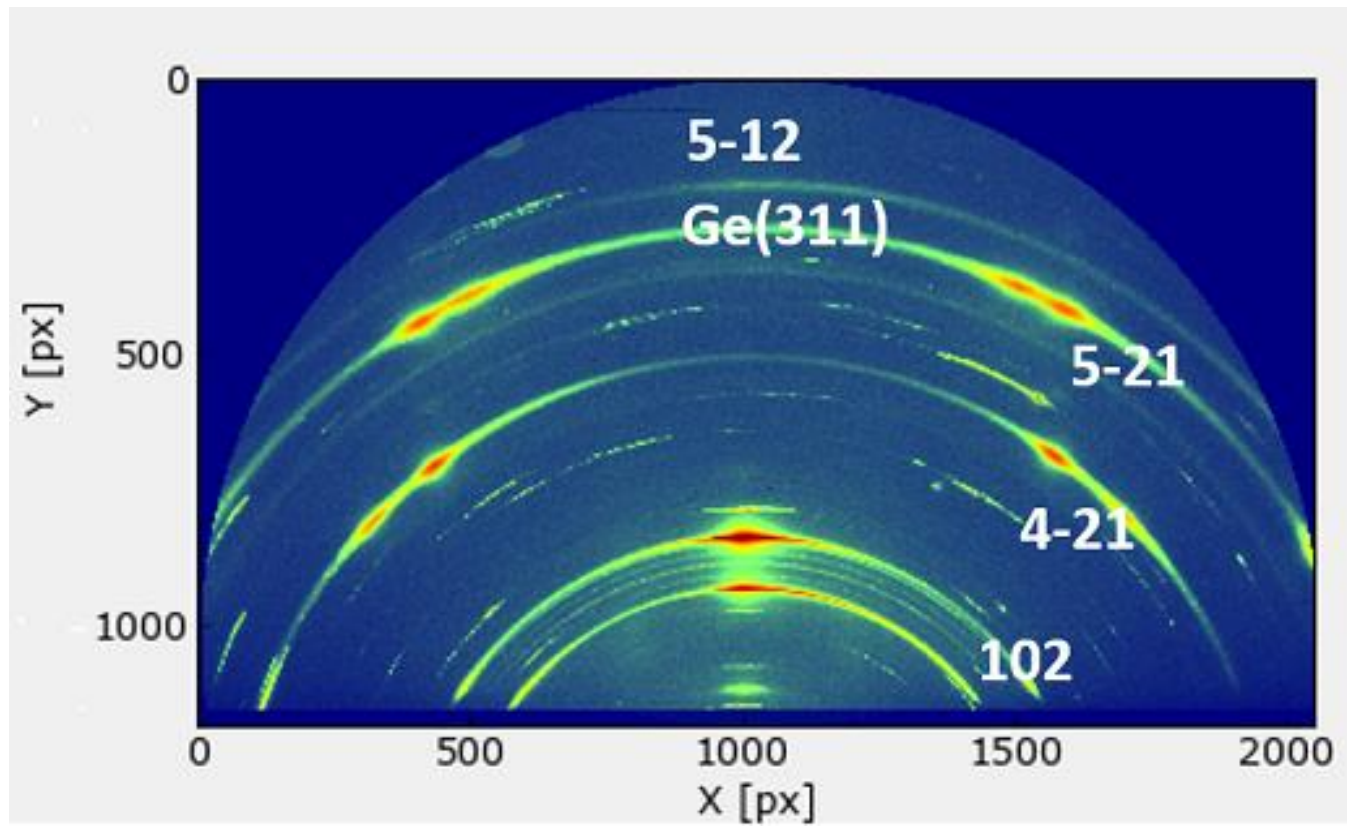

Figure 1. 2D-GIXRD for a simple with carbon, where the indexed Debye rings belong to the Mn5Ge3.
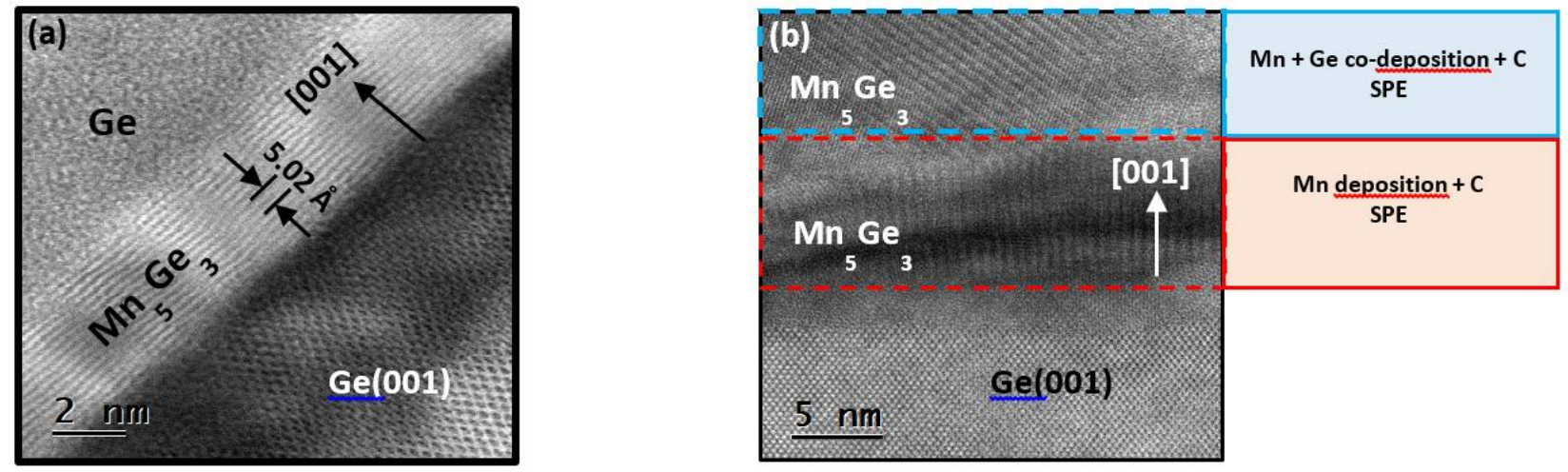

Figure 2. HR-TEM micrographs of Mn5Ge3 samples: (a) without carbon and (b) with carbon.

\section{References}

[1] A. Spiesser, H. Saito, R. Jansen, S. Yuasa, K. Ando, Large spin accumulation voltages in epitaxial Mn5Ge3 contacts on Ge without an oxide tunnel barrier, Phys. Rev. B 90 (2014) 205213.

[2] O. Abbes, A. Portavoce, V. Le Thanh, C. Girardeaux, L. Michez, Phase formation during Mn thin film reaction with Ge: Self-aligned germanide process for spintronics, Appl. Phys. Lett. 103 (2013) 172405. [3] C. Zeng, S.C. Erwin, L.C. Feldman, A.P. Li, R. Jin, Y. Song, J.R. Thompson, H. H. Weitering, Epitaxial ferromagnetic Mn5Ge3 on Ge(111), Appl. Phys. Lett. 83 (2003) 5002. 